\title{
Determining the Spatial Coherence of Excitons from the Photoluminescence Spectrum in Charge-Transfer J-aggregates
}

\author{
Nicholas J. Hestand and Frank C. Spano \\ Department of Chemistry \\ Temple University \\ Philadelphia, Pennsylvania, 19122, USA
}

\begin{abstract}
The importance of spatial coherence in energy and charge transfer processes in biological systems and photovoltaic devices has been hotly debated over the past several years. While larger spatial coherences are thought to benefit transport, a clear correlation has yet to be established, partly because a simple and accurate measure of the coherence length has, for the most part, remained elusive. Previously, it was shown that the number of coherently connected chromophores, $N_{C o h}$, can be determined directly from the ratio $\left(S_{R}\right)$ of the $0-0$ and $0-1$ vibronic line strengths in the photoluminescence (PL) spectrum. The relation $N_{C o h}=\lambda_{0}^{2} S_{R}$, where $\lambda_{0}^{2}$ is the associated Huang-Rhys parameter, was derived in the Frenkel exciton limit. Here, it is shown that $S_{R}$ remains a highly accurate measure of coherence for systems characterized by significant charge transfer interactions (e.g. conjugated $\pi$-stacked systems). The only requirement is that the exciton band curvature must be positive, as in a J-aggregate.
\end{abstract}

\section{Introduction}

The involvement of coherence in exciton and charge transport is a hotly debated topic in the fields of materials science and biology especially with regards to the operation of organic photovoltaic devices [1-7] and light harvesting in photosynthetic antenna systems.[8-15] Interest in coherence stems from its possible role in enhancing exciton and charge transport -bAs one important example, allistic transport (vs. diffusive hopping) could dramatically enhance the efficiency of solar cells by extending the range over which an exciton can travel before recombining. Accurately measuring the coherence length - the length over which the exciton or 
charge behaves like a wave - is a vital first step to understanding the general role coherence plays in transport.

The number of coherently coupled chromophores, $N_{c o h}$, has been estimated from the superradiative enhancement of the excited state decay rate [16-22], analysis of the inhomogeneous linewidth of the excitonic absorption spectrum [23,24] and from the peak-to-dip spectral separation in the transient absorption spectrum.[15,25-27] However, all such methods neglect the important role of vibronic coupling in the $S_{0} \rightarrow S_{1} \mathrm{UV}$-Vis transition from which the exciton band is derived. Such coupling gives rise to the pronounced vibronic progressions typical of many $\pi$-conjugated molecules which readily form aggregates, such as the celebrated cyaninebased J-aggregates.[28-30] Vibronic coupling acts to narrow the exciton bandwidth, thereby reducing the coherence length, and making energy and charge transport more susceptible to defects.

Recently, we presented a straightforward method to determine $N_{c o h}$ for excitons directly from the steady state photoluminescence (PL) spectral line shape which accurately accounts for vibronic coupling.[31,32] The method derives from aggregation-induced changes to the vibronic progression in the PL spectrum. In particular, the ratio of the $0-0$ to $0-1$ line strengths in the steady-state PL spectrum of J-aggregates, or any aggregated system with a positive exciton band curvature, is closely approximated by $N_{c o h} / \lambda_{0}^{2}$, where $\lambda_{0}^{2}$ is the Huang-Rhys (HR) factor characterizing the monomer PL spectrum. This simple expression was first derived for Frenkel excitons in a site-disordered lattice using the Holstein-style Hamiltonian to account for vibronic coupling. In the limit of disorder-free aggregates at low temperature the coherence number reduces to the actual number of chromophores defining the aggregate, $N$. In this limit, the $0-0 / 0-1$ line strength ratio is rigorously equal to $N / \lambda_{0}^{2}$, independent of the exciton bandwidth, HR factor or vibrational energy.[31] Remarkably, the PL ratio remains an invariant over the entire phase space defining the Frenkel-Holstein Hamiltonian. As a point of comparison, the superradiant enhancement of the radiative decay rate is given by $F N_{c o h}$, where $F$ is a generalized FranckCondon factor ranging from $\exp \left(-\lambda_{0}^{2}\right)$ in the weak exciton coupling limit to $\exp \left(-\lambda_{0}^{2} / N\right)$ in the strong coupling limit [31]. Hence, extracting the coherence number using superradiance requires a priori knowledge of the exciton coupling strength.Moreover, measuring the aggregate radiative decay rate is complicated by the presence of nonradiative decay mechanisms, thereby requiring 
knowledge of the temperature-dependent quantum yield. Such measurements are difficult to make, especially for crystalline samples.

In a more recent development we showed that the PL ratio can also be used to accurately track the temporal change in $N_{\text {coh }}$ following impulsive excitation. [32] Shortly thereafter, Sung et al. demonstrated the idea experimentally by utilizing time-resolved PL to monitor subpicosecond exciton localization in perylene diimide aggregates [33]. The PL ratio has also been used to justify the ultra-large coherence lengths measured in practically defect-free polyacetylene chains, also referred to as quantum wires [34,35]; to determine $N_{c o h}$ in polyacenes crystals [31,36] and dinaptho-thieno-thiophene (DNTT) crystals [37]; and to monitor coherence in a phosphorescent Pt-containing polymer.[38]

In this work we extend our analysis of the PL ratio to include aggregates with significant intermolecular charge transfer. This is particularly relevant for $\pi$-stacked geometries where nearest neighbors are typically less than 4 Angstroms apart. At such close distances significant nearest neighbor wave function overlap promotes Frenkel exciton dissociation into charge separated states. The mixing between Frenkel and charge-transfer (CT) excitons has been extensively studied in a variety of rylene-based crystals and aggregates [39-48] where CT states play an important role in determining photophysical and transport properties. There has also been a renewed interest in CT states in polyacene crystals [49-53] due to their likely involvement in singlet fission.[54] In Ref. [55] we showed that Frenkel/CT mixing can lead to the formation of unconventional $\mathrm{J}$ and $\mathrm{H}$-aggregates where the exciton coupling arises from a superexchange mechanism involving electron or hole transfer through an intermediate virtual CT state. Such aggregates display the same vibronic signatures as conventional Kasha $\mathrm{H}$ and J-aggregates[56], even in the absence of Coulombic coupling.[46,55]

In what follows we utilize a Holstein-style Hamiltonian to investigate PL in $\pi$-stacked aggregates. The Hamiltonian is expanded over that used in Ref. [31] to include Frenkel/CT exciton mixing. As such we include the HR factors, which are closely related to Marcus reorganization energies, for the neutral excited species (Frenkel exciton) as well as for charged molecules hosting an extra electron or hole. We show that the PL ratio formula derived in Ref. [31] can be simply modified to account for CT and is applicable to any aggregate in which the exciton band has positive curvature, as in J-aggregates. We further include the effect of 
increasing temperature, showing that the PL ratio can be employed to accurately measure the thermal coherence length in molecular $\pi$-stacks.

\section{Hamiltonian for 1 -D $\pi$-stacks}

The photophysical properties of $\pi$-stacks of conjugated chromophores are well described by a Holstein-like Hamiltonian that considers both Frenkel and charge-transfer (CT) excitations coupled nonadiabatically to an effective local vibrational mode with energy $\hbar \omega_{v i b} \approx 1400 \mathrm{~cm}^{-1}$. The relevant vibrational mode (or group of modes) is associated with the nuclear displacement that occurs upon excitation as the molecular geometry adjusts to the new electron density of the excited state. Upon electronic excitation, the double bonds of the chromophore lengthen while single bonds shorten. If aromatic groups are involved the geometry becomesmore quinoidal and less aromatic. This coupling of the nuclear and electronic degrees of freedom gives rise to the pronounced vibronic progressions observed in many $\pi$-conjugated molecules. Such progressions provide signatures of J- and $\mathrm{H}$-aggregation[57], and as we show here, a measure of the exciton's spatial coherence in aggregates with a positive band curvature.

Assuming periodic boundary conditions and working within the subspace of a single electron and a single hole, the Holstein-like Hamiltonian for a 1-D $\pi$-stack is given in the delocalized exciton-phonon basis as

$$
\begin{aligned}
H & =E_{S_{1}}+\sum_{k, s}\left[J_{k} \delta_{s, 0}+V_{C T}(s)\left(1-\delta_{s, 0}\right)\right]|k, s\rangle\langle k, s|+\sum_{s, k}\left\{\left(t_{e}+t_{h} e^{i k}\right)|k, s\rangle\langle k, s+1|+H . c .\right\} \\
& +h \omega_{v i b} \sum_{q} b_{q}^{\dagger} b_{q}+\frac{\hbar \omega_{v i b}}{\sqrt{N}} \sum_{k, q, s}\left\{\left[\lambda_{0} \delta_{s, 0}+\left(\lambda e^{i q s}+\lambda_{+}\right)\left(1-\delta_{s, 0}\right)\right]|k, s\rangle\langle k+q, s| b_{q}^{\dagger}+H . c .\right\} \\
& +\hbar \omega_{v i b} \sum_{k, s}\left[\lambda_{0}^{2} \delta_{s, 0}+\left(\lambda_{-}^{2}+\lambda_{+}^{2}\right)\left(1-\delta_{s, 0}\right)\right]|k, s\rangle\langle k, s| .
\end{aligned}
$$

where the ground electronic/vibrational state is taken as the zero of energy. The electronic part of the Hamiltonian is identical to the one used by Merrifield in Ref. [58] and similar Hamiltonians (including vibronic coupling) have been used to investigate the low lying excited states of acene aggregates [51,52,59], and rylene-based aggregates [39-48]. In Eq. (1) the electronic states are defined in terms of their wave vector, $k=2 \pi l / N(l=0, \pm 1, \pm 2, \ldots, N / 2)$ (where $N$ is taken to be 
even), and the local separation between electron and hole, $s(=0, \pm 1, \pm 2 \ldots, N / 2)$. The delocalized excitons can be expanded in terms of local excitons as,

$$
|k, s\rangle=\frac{1}{\sqrt{N}} \sum_{n} e^{i k n}|n, n+s\rangle,
$$

where $n$ runs from 1 to the total number of molecules in the aggregate, $N$. The ket $|n, n+s\rangle$ represents the electronically excited state where molecule $n$ hosts a hole and molecule $n+s$ hosts an electron. Hence, $|n, n\rangle$ defines a localized Frenkel exciton where the electron and hole reside on the same chromophore while $|n, n+s\rangle(s \neq 0)$ defines a CT exciton where the electron and hole reside on different chromophores.

In Eq. (1), the energy of a local Frenkel exciton is defined relative to the ground electronic and vibrational state as $E_{S_{1}}$. Furthermore, the energy of a delocalized Frenkel exciton with wave vector $k$ (prior to mixing with the CT states, i.e. diabatic), is given by $E_{S_{1}}+J_{k}$, where the $k$ th Fourier component of the Coulomb coupling is,

$$
J_{k}=\frac{1}{N} \sum_{n, m} J_{n, m} e^{i k(n-m)} .
$$

$J_{n, m}$ is the Coulombic coupling between the $n$th and $m$ th chromophores. The energies of the diabatic CT excitons, $E_{S_{1}}+V(s)$, depend on the separation between electron and hole due to the Coulomb binding potential. For simplicity we restrict the electron and hole to nearest neighbors by defining,

$$
\begin{aligned}
V(s) & =E_{C T}-E_{S_{1}} & & |s|=1 \\
& =\infty & & |s|>1
\end{aligned}
$$


where $E_{C T}$ is the energy of a nearest neighbor CT state. $E_{C T}$ can be decomposed as $E_{C T} \equiv I_{P}-E_{A}+E_{P}-e^{2} / 4 \pi \varepsilon_{0} \varepsilon_{r} d$ where $d$ is the distance between nearest neighbor chromophores, $\varepsilon_{0}$ and $\varepsilon_{r}$ are the vacuum and relative permittivities, and $I_{P}, E_{A}$, and $E_{P}$ are the ionization potential, electron affinity and polarization energy, respectively. The nearest neighbor approximation given by Eq. (4) expedites numerically solving Eq. (1) without dramatically affecting the accuracy of the results, as long as the non-nearest neighbor CT states are sufficiently high in energy i.e. the Coulomb potential well is larger than the electron and hole bandwidths. Finally, the electron and hole transfer integrals, $t_{e}$ and $t_{h}$ facilitate charge migration throughout the system by coupling neighboring LUMOs $\left(t_{e}\right)$ and neighboring HOMOs $\left(t_{h}\right)$.

In this work the phonons which result from delocalized intramolecular vibrations are considered to be dispersionless, i.e. Einstein phonons. The phonon operators $b_{q}^{\dagger}$ and $b_{q}$ in Eq. (1) create and annihilate phonons having wave vector $q=2 \pi l / N(l=0, \pm 1, \pm 2, \ldots, N / 2)$. The phonon operators can be expressed in terms of local phonon operators as

$$
b_{q}^{\dagger}=\frac{1}{\sqrt{N}} \sum_{n}^{i q n} b_{n}^{\dagger},
$$

where $b_{n}^{\dagger}$ creates a vibrational excitation on molecule $n$. The final three summations in Eq. (1) define the vibrational energy, the vibronic coupling, and the relaxation energy, respectively. The magnitudes of the vibronic couplings and relaxation energies are determined by the neutral HR parameter, $\lambda_{0}^{2}$, and the ionic HR parameters for anionic and cationic molecules, $\lambda_{-}^{2}$ and $\lambda_{+}^{2}$, respectively. Note that in contrast to Ref.[31], the totally symmetric phonon $(q=0)$ is not generally separable from the electronic states due to the non-equivalent HR factors for the Frenkel and CT excitons. (An important exception is the case when $\lambda_{-}+\lambda_{+}=\lambda_{0}$, see Appendix A.) Hence, the number of totally symmetric phonons is not a good quantum number of Eq. (1) as it is in the Frenkel limit when CT excitons are neglected.

The Hamiltonian in Eq. (1) can be represented in a product basis of electronic and vibrational excitations which are expressed in bra-ket notation as $\left|k, s ; n_{q_{0}}, n_{q_{1}}, n_{q_{2}}, \ldots\right\rangle$. Here, $k$ 
and $s$ determine the wave vector of the electronic state and the separation between the electron and hole respectively, while the $n_{q_{l}}$ denote the number of phonons characterized by wave vector $q_{l}=2 \pi l / N$ in the ground state nuclear potential well. Since we have assumed periodic boundary conditions, the total momentum $\kappa=k+n_{q_{1}} q_{1}+n_{q_{2}} q_{2}+\ldots$ (modulo $\left.2 \pi\right)$ is a good quantum number. The eigenstates of the Hamiltonian can therefore be characterized by $\kappa$ and expressed as,

$$
\left|\Psi_{\kappa, \alpha}\right\rangle=\sum_{k, s} \sum_{n_{q_{0}}, n_{q_{1}}, n_{q_{2}}, \ldots}^{\prime} c_{k, s, n_{q_{0}}, n_{q_{1}}, n_{q_{2}}, \ldots}^{\kappa, \alpha}\left|k, s ; n_{q_{0}}, n_{q_{1}}, n_{q_{2}}, \ldots\right\rangle .
$$

In Eq. (6) the prime over the second summation indicates that the number of phonons are restricted to values that conserve the total momentum $\kappa$ for a given $k$. Moreover $\alpha(=1,2, \ldots)$ labels the eigenstates of a given $\kappa$ in order of increasing energy, where the energy corresponding to the state $\left|\Psi_{\kappa, \alpha}\right\rangle$ is $\hbar \omega_{\kappa, \alpha}=\left\langle\Psi_{\kappa, \alpha}|H| \Psi_{\kappa, \alpha}\right\rangle$.

An important feature of Eq. (1) is that it permits mixing among Frenkel and CT excitons through the charge transfer integrals. This allows for unique phenomena such as CT mediated Jand $\mathrm{H}$ - aggregates where the exciton band curvature and associated spectral signatures are determined predominantly by the Frenkel/CT interactions.[46-48,55] The magnitude of FE/CT mixing between a Frenkel and CT state with a given $k$ is determined primarily by the energy difference $E_{C T}-E_{S_{1}}-J_{k}$, and the magnitude of the FE/CT coupling, $\left|t_{e}+t_{h} e^{i k}\right|$. In the current manuscript, we are specifically interested in CT mediated J-aggregates, which require $t_{e} t_{h}>0$ (using translational symmetry to define phase) so that the totally symmetric $(\kappa=0)$ exciton lies at the bottom of the exciton band [55] as demonstrated in Fig. 1. To simplify our parameter space we therefore set $t_{e}=t_{h} \quad\left(=\hbar \omega_{v i b}\right)$ for all simulations that follow. Furthermore, we set the Coulomb coupling to zero $\left(J_{k}=0\right)$ so that the Frenkel/CT mixing can be adjusted by simply changing $E_{C T}-E_{S_{1}}$. We emphasize, however, that our results are valid as long as the band curvature is positive - i.e. J-like - even in the presence of Coulombic coupling.[46] 


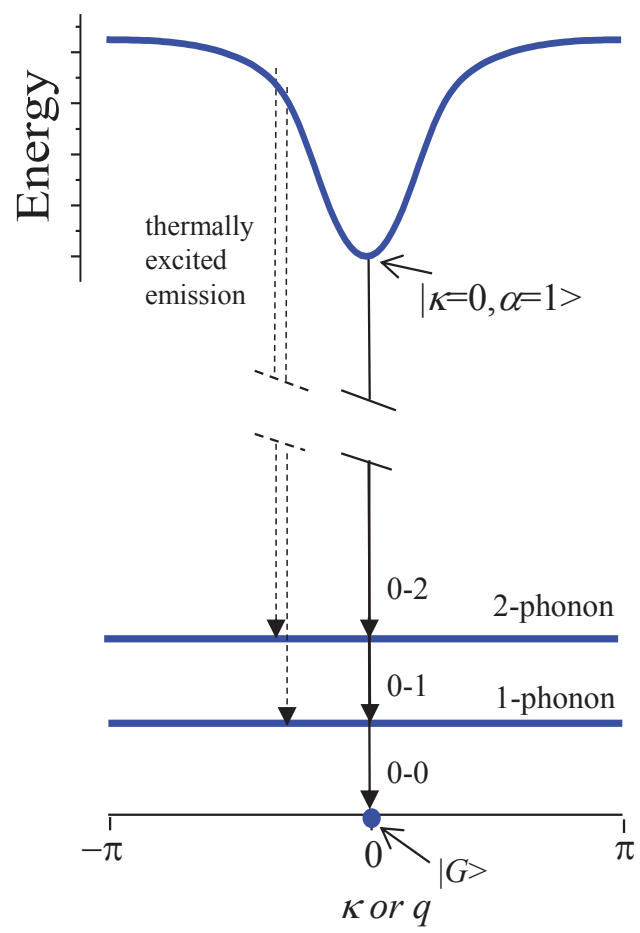

Figure 1: Emission from an exciton band with positive curvature (J-like). The 0-0 emission terminates on the vibrationless electronic ground state $\mid \mathrm{G}>$ and is allowed only from the state at the band bottom $(\kappa=0, \alpha=1)$. By contrast, the $0-v(v>1)$ emission is allowed from all states, regardless of $\kappa$ as long as momentum is conserved. For example, in 0-1 emission the wave vector of the emitting exciton $\kappa$ must match the wave vector of the terminal phonon, $q$. All phonons are assumed to be dispersionless (Einstein phonons).

\section{Photoluminescence spectrum of CT mediated J-aggregates at low temperature}

Once the coefficients of Eq. (6) are known, the reduced photoluminescence (PL) spectrum can be calculated according to

$$
S(\omega, T)=\sum_{\kappa, \alpha} \sum_{v} P_{\kappa, \alpha}(T) I_{\kappa, \alpha}^{0-v} \Gamma\left(\omega-\omega_{\kappa, \alpha}+v \omega_{v i b}\right)
$$

$S(\omega, T)$ is referred to as the reduced PL spectrum because we have omitted the cubic frequency dependence in the emission transitions in order to focus solely on the line strengths. In Eq. (7), $I_{\kappa, \alpha}^{0-v}$ is the line strength corresponding to the transition from the excited state $\Psi_{\kappa, \alpha}$, to the ground electronic state with $v$ vibrational excitations. The line strengths for such transitions are determined from 


$$
I_{\kappa, \alpha}^{0-v}=\mu^{-2} \sum_{n_{q_{0}}, n_{q_{1}}, n_{q_{2}} \ldots, n_{q_{N-1}}}^{\prime}\left|\left\langle\Psi_{\kappa, \alpha}|\hat{\mu}| g ; n_{q_{0}}, n_{q_{1}}, n_{q_{2}}, \ldots, n_{q_{N-1}}\right\rangle\right|^{2}
$$

where the prime on the summation indicates that the total number of ground state phonons is $v$. The state $\left|g ; n_{q_{0}}, n_{q_{1}}, n_{q_{2}}, \ldots, n_{q_{N-1}}\right\rangle$ represents the ground electronic state accompanied by $n_{q_{l}}$ phonons with wave vector $q_{l}$. Furthermore, $\hat{\mu}$ is the transition dipole moment operator with matrix elements $\langle k, s|\hat{\mu}| g\rangle=\sqrt{N} \mu \delta_{k, 0} \delta_{s, 0}$. Analysis of Eq. (8) shows that only Frenkel excitons with $\kappa=0$ - most notably the state at the band bottom $(\kappa=0, \alpha=1)$ - can source $0-0$ emission, i.e. only these states can radiate to the vibrationless ground state. Side-band emission has no such restriction - any state can radiate to a vibrationally excited ground state so long as the total momentum is conserved. For example, $0-1$ emission from state $\kappa, \alpha=1$ can terminate on a phonon with wave vector $q=\kappa$. Fig. 1 illustrates these selection rules.

The temperature dependence of the PL spectrum arises from a thermal average over a Boltzmann distribution of emitting excitons. The thermal population $P_{\kappa, \alpha}(T)$ is given by,

$$
P_{\kappa, \alpha}(T)=\frac{1}{Z(T)} \exp \left(-\hbar \omega_{\kappa, \alpha} / k_{b} T\right)
$$

where $Z(T)=\sum_{\kappa, \alpha} \exp \left[-\hbar \omega_{\kappa, \alpha} / k_{b} T\right]$ is the partition function. In the low temperature limit $k_{b} T$ is much less than the difference in energy between the lowest two excitons at the bottom of the exciton band. The distribution then reduces to $P_{\kappa, \alpha}(T)=\delta_{\kappa, 0} \delta_{\alpha, 1}$ for J-aggregates. Finally, $\Gamma(\omega)$ in Eq. (7) is a line shape function which is taken to be a normalized Gaussian. In what follows we define the ratio of the $0-0$ to $0-1$ PL line strengths for a temperature $T$ as,

$$
S_{R}(T) \equiv \frac{\left\langle I_{\kappa, \alpha}^{0-0}\right\rangle_{T}}{\left\langle I_{\kappa, \alpha}^{0-1}\right\rangle_{T}}
$$




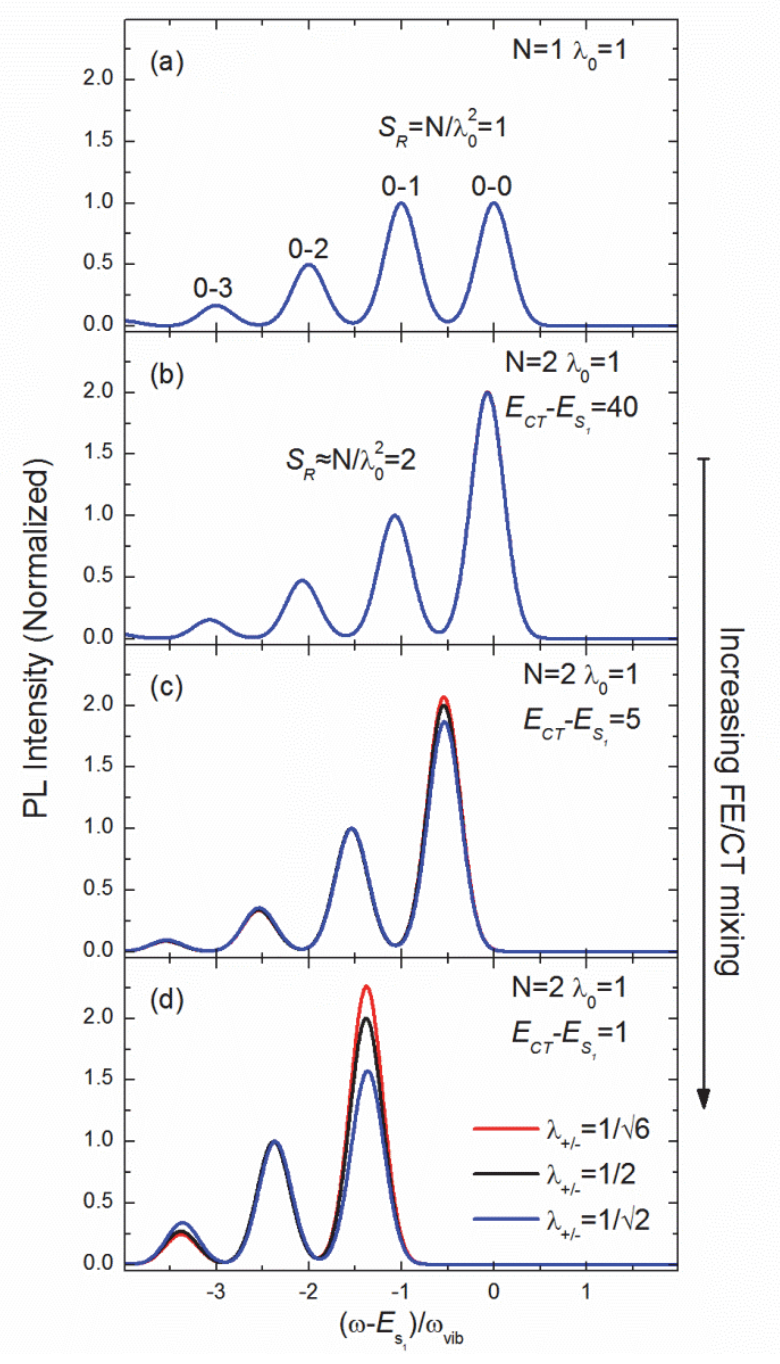

Figure 2. The PL spectrum for monomers (a) and CT mediated J-aggregate dimers (b)-(d) at $T=0 \mathrm{~K}$ for select values of $\lambda_{+/-}$. The parameters $\lambda_{0}=1, J_{k}=0$ and $t_{e}=t_{h}=1$ are common to all simulations where all energies are expressed in units of vibrational quanta. For the dimers, the FE/CT mixing is controlled by the resonance between the diabatic FE and CT states as determined by $E_{C T}-E_{S_{1}}$ (see insets for values). When there is little FE/CT mixing (b), $S_{R}$ is given approximately by $N / \lambda_{0}^{2}$ for all values of $\lambda_{+/-}$. As the FE/CT mixing increases $(\mathrm{c}, \mathrm{d})$, the ratio begins to diverge from $N / \lambda_{0}^{2}$ depending on the values of $\lambda_{+/-}$. All spectra have been normalized to the intensity of the $0-1$ peak in order to emphasize $S_{R}$.

Here, $\langle\ldots\rangle_{T}$ denotes a Boltzmann average. Further details of the thermally averaged emission can be found in Ref.[31]

Fig. 2(a) shows the calculated PL spectrum for a monomer when $\lambda_{0}^{2}=1$, an approximate value for the $\sim 1400 \mathrm{~cm}^{-1}$ vibrational mode in many conjugated molecules. In the monomer limit, the $0-v$ transition strengths are proportional to $I^{0-v}=\lambda_{0}^{2 v} e^{-\lambda_{0}^{2}} / v$ !, so that the PL ratio is simply $S_{R}=1 / \lambda_{0}^{2} \cdot S_{R}$ reduces to 1 for the monomers in Fig. 2(a), as manifest by equal 0-0 and 0-1 peak intensities.

In aggregates, deviations of the ratio from the monomeric limit provide signatures of the exciton coherence length, which generally depends on number of chromophores as well as the strength of the intermolecular interactions, the degree of disorder, and the temperature.[31] At 
low temperatures, the behavior of $S_{R}$ is unique for J-aggregates (or any aggregate with a positive exciton band curvature). In Ref. [31] it was shown that for Frenkel excitons at low temperatures and in the absence of disorder,

$$
S_{R}(T \rightarrow 0 K)=N / \lambda_{0}^{2} \quad t_{e}=t_{h}=0
$$

which can be obtained using the Hamiltonian in Eq. (1) in the limit that $t_{e}$ and $t_{h}$ approach zero (i.e. no Frenkel/CT exciton coupling). This result is remarkable in that it is rigorous over the entire parameter space $\left(\omega_{v i b}, \lambda^{2}, J_{k}\right)$ of the Holstein Hamiltonian for J-aggregates, or, in fact, any aggregate with an allowed 0-0 transition from the lowest energy exciton. However, in many aggregate systems short ( $\sim 4 \AA) \pi$-stacking distances facilitate significant $\mathrm{CT}$ interactions that result in substantial mixing between Frenkel and CT excitons. In these systems, Frenkel/CT interactions must be considered in order to accurately describe the low energy photophysics. [3948,55] Under these conditions, the ratio formula developed in Ref.[31] needs to be reconsidered.

In Fig.'s 2(b)-(d) the low-temperature PL spectrum of CT mediated J-aggregate dimers is shown as a function of increasing Frenkel/CT mixing. The spectrum was evaluated from the Hamiltonian in Eq. (1) expressed in a basis set of all one- and two-particle states with a cap on the total number of vibrational quanta equal to four.[60] Note that for a dimer the sum of all oneand two-particle states forms a complete basis set within the framework of our model (i.e. there are no three- and higher-particle states); hence we are able to obtain essentially exact results for low energy excitations. In Fig.'s 2(b)-(d), the neutral HR factor was set to unity, so that in the limit of non-interacting chromophores the PL spectrum reduces to that shown in Fig. 2(a). In going from (b) to (d), the Frenkel/CT interaction is enhanced as the diabatic CT excitons are lowered into resonance with the Frenkel excitons. In the Frenkel limit (Fig. 2(b)), the ratio is $N / \lambda_{0}^{2}(=2)$ consistent with Ref. [31]. However, as the Frenkel and CT excitons begin to mix significantly (Fig. 2(c),(d)), the ratio deviates from $N / \lambda_{0}^{2}$ in a manner that depends on the values of the ionic Huang-Rhys parameters, $\lambda_{+}$and $\lambda_{-}$. When the sum,$\left(\lambda_{+}+\lambda_{-}\right)^{2}$, exceeds the HR factor for a neutral molecule $\lambda_{0}^{2}$, the ratio decreases relative to the Frenkel result in Eq. (11), 

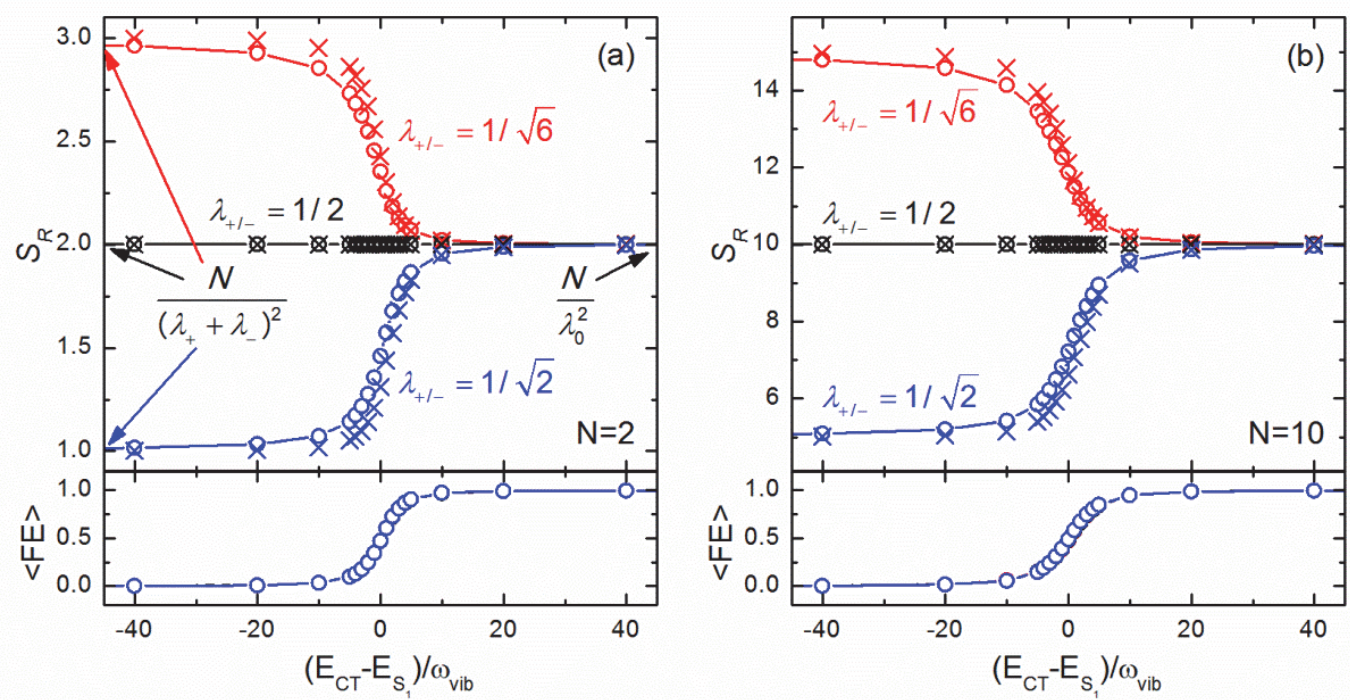

Figure 3. The low-temperature PL ratio for CT mediated J-aggregates as a function of $E_{C T}-E_{S_{1}}$ for $N=2$ (a) and $N=10$ (b) are shown in the top panels. Note the different vertical scales in (a) and (b). Open circles represent numerical results while the data points represented as crosses are the solution to Eq. (12) where $\langle F E\rangle$ (bottom panels) is calculated numerically. The parameters $\lambda_{0}=1, J_{k}=0$ and $t_{e}=t_{h}=1$ are common to all simulations where all energies are expressed in units of vibrational quanta. When $E_{C T}-E_{S_{1}} \gg 0$, the emitting state is dominated by Frenkel character and $S_{R}$ approaches $N / \lambda_{0}^{2}$. When $E_{S_{1}}-E_{C T} \gg 0$ the emitting state is dominated by CT character and $S_{R}$ approaches $N /\left(\lambda_{+}+\lambda_{-}\right)^{2}$. Interestingly, when $\lambda_{+}+\lambda_{-}=\lambda_{0}$ the ratio is constant for all values of $E_{C T}-E_{S_{1}}$.

while the reverse holds when $\left(\lambda_{+}+\lambda_{-}\right)^{2}<\lambda_{0}^{2}$. Furthermore, when $\left(\lambda_{+}+\lambda_{-}\right)^{2}=\lambda_{0}^{2}$ the ratio remains equal to that obtained in the Frenkel limit. (Fig.2, when $\lambda_{+}=\lambda_{-}=1 / 2$ )

To investigate this behavior further, we plot in Fig. 3(a) $S_{R}$ as a function of $E_{C T}-E_{S_{1}}$ (open circles) for the CT-mediated J-aggregate dimers. The character of the emitting state is determined by the relative energies of the diabatic Frenkel and CT excitons. When $E_{C T}>E_{S_{1}}$ the emitting state - or state with lowest energy - is primarily a Frenkel exciton. Conversely, when $E_{C T}<E_{S_{1}}$, the emitting state has a majority CT character. In the limit of a Frenkel dominated emitting state $\left(E_{C T}-E_{S_{1}}>>\left|t_{e}\right|,\left|t_{h}\right|\right)$, the ratio approaches $N / \lambda_{0}^{2}$. Interestingly, in the opposite limit of a CT dominated emitting state, $E_{S_{1}}-E_{C T}>>\left|t_{e}\right|,\left|t_{h}\right|$, the ratio approaches $N /\left(\lambda_{+}+\lambda_{-}\right)^{2}$. Fig. 3(b) shows that the same limiting behavior is observed in larger aggregates where the ratio 
of a CT mediated J-aggregate ten-mer $(N=10)$ is plotted as a function of $E_{C T}-E_{S_{1}}$. To evaluate the spectra for $N=10$ we utilized the three-particle approximation, described in greater detail in Appendix B.

Based on the limiting behavior of the ratio as a function of the FE/CT mixing and the result of Ref.[31], we propose that at low temperatures and in the absence of disorder, $S_{R}$ can be approximated by

$$
S_{R}(T \rightarrow 0 K) \approx \frac{N}{\langle F E\rangle \lambda_{0}^{2}+(1-\langle F E\rangle)\left(\lambda_{+}+\lambda_{-}\right)^{2}},
$$

where $\langle F E\rangle$ is the Frenkel component of the emitting (lowest energy) state,

$$
\langle F E\rangle=\sum_{k}\left|\left\langle\Psi_{\kappa=0, \alpha=1} \mid k, s=0\right\rangle\right|^{2}
$$

Fig. 3 shows that Eq. (12) (crosses) does an excellent job of reproducing the numerically calculated ratio.

Eq. (12) verifies the observations made in Fig 3: when $\left(\lambda_{+}+\lambda_{-}\right)^{2}<\lambda_{0}^{2}$, the ratio increases relative to the Frenkel limit as the $\mathrm{CT}$ admixture of the emitting state increases, while the opposite occurs when $\left(\lambda_{+}+\lambda_{-}\right)^{2}>\lambda_{0}^{2}$. Interestingly, when $\lambda_{+}+\lambda_{-}=\lambda_{0}$, Eq. (12) predicts that the ratio will remain constant regardless of the CT admixture within the emitting state. This prediction is confirmed in Fig. 3 for the case $\lambda_{+}=\lambda_{-}=\lambda_{0} / 2(=1 / 2)$. In fact, it can be rigorously shown (see Appendix A) that when $\lambda_{+}+\lambda_{-}=\lambda_{0}$, one recovers the result obtained for pure Frenkel excitons, i.e.

$$
\lim _{\lambda_{+}+\lambda_{-} \rightarrow \lambda_{0}} S_{R}(T \rightarrow 0 K)=N / \lambda_{0}^{2}
$$

at low temperature and in the absence of disorder. This result is exact for any aggregate described by the Hamiltonian in Eq. (1), i.e. for any value of Coulomb coupling, any level of 


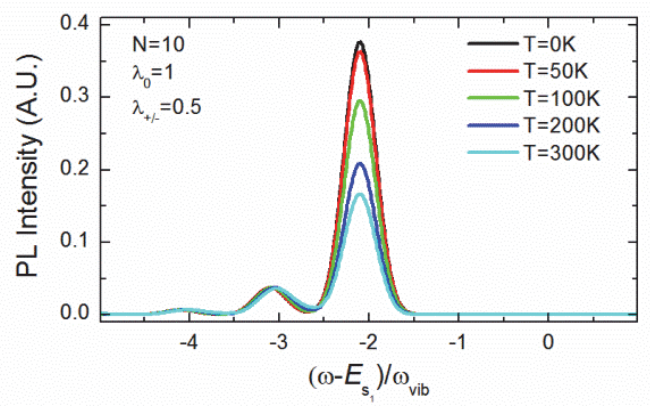

Figure 4. The PL spectrum for a CT mediated J-aggregate with $N=10$ chromophores at various temperatures as denoted in the legend. The system parameters are $\lambda_{0}=1, \lambda_{+}=\lambda_{-}=$ $0.5, J_{k}=0, t_{e}=t_{h}=1$, and $E_{C T}-E_{S_{1}}=1$ where all energies are given in units of vibrational quanta. As the temperature increases, the PL ratio decreases. Note the insensitivity of the $0-1$ peak to temperature. The spectra have not been normalized.

Frenkel/CT mixing, any vibrational energy $\hbar \omega_{v i b}$, any value of $\lambda_{0}^{2}$ and any number of chromophores $N$. The only requirement is that the exciton band curvature remains positive, as for a J-aggregate.

\section{PL spectrum at Higher Temperatures}

In Section III, we considered emission from ordered CT mediated J-aggregates at low temperatures so that emission originated only from the lowest energy state, $\Psi_{\kappa=0, \alpha=1}$. We now consider the case of higher temperatures where higher energy "hot" excitons are populated thermally. Fig. 4 shows how the PL spectrum of a CT mediated J-aggregate with $N=10$ changes as the temperature increases. While the $0-1$ and higher vibronic peaks remain relatively stable over the entire range of temperatures [31], the 0-0 peak drops significantly with increasing temperature. The decrease is due to the depopulation of the state $\Psi_{\kappa=0, \alpha=1}$ with increasing temperature, as detailed in Ref. [31].

In Ref. [31] it was shown that for Frenkel mediated J-aggregates $S_{R}$ is a signature of the exciton's coherence. This arises because the 0-0 intensity results from a coherent sum over all molecular transition dipoles, whereas the sideband emission (i.e. 0-1, 0-2, .. emission) results from an incoherent dipole sum. Furthermore, it was shown that the ratio formula $\left(S_{R}=N / \lambda_{0}^{2}\right)$ can be modified to reflect the localizing influences of disorder and increasing temperature by simply replacing $N$ with the coherence number $N_{c o h}$. In the case of increasing temperature, emission arises from a Boltzmann distribution of emitting states. An average over such a distribution results in a diminishing thermal coherence number, $N_{c o h}(T)<N$. Our goal in this 

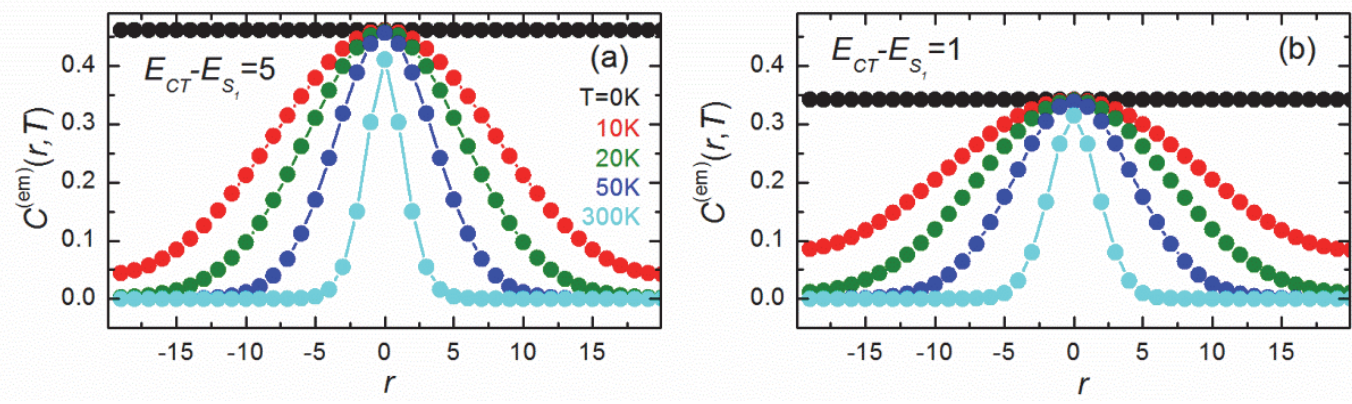

Figure 5. The thermal coherence function of the emitting state at various temperatures for CT mediated J-aggregates with $N=40$ chromophores. For (a) $E_{C T}=E_{S_{1}}=5$ and (b) $E_{C T}=E_{S_{1}}=$ 1. The remaining system parameters are given by $\lambda_{0}=1, \lambda_{+}=\lambda_{-}=\sqrt{2} / 2, J_{k}=0$, and $t_{e}=$ $t_{h}=1$ where all energies are given in terms of vibrational quanta. As temperature increases, the coherence function collapses about $r=0$ indicating a decreasing in the coherence number, $N_{C o h}$.

section is to show that the PL ratio remains an indicator of the exciton coherence number in CT mediated J-aggregates.

In line with Ref. [31], the exciton coherence function is defined as,

$$
C^{(e m)}(r, T)=\sum_{\kappa, \alpha} P_{\kappa, \alpha}(T)\left\langle\Psi_{\kappa, \alpha}\left|\left(\sum_{n}|n, n ; v a c\rangle\langle n+r, n+r ; v a c|\right)\right| \Psi_{\kappa, \alpha}\right\rangle,
$$

where $|n, n ; v a c\rangle$ represents the state in which a local Frenkel excitation resides on molecule $n$ with all molecules in their vibrational ground states ( $v a c$ ) characterized by the electronic ground state nuclear geometry. Fig. 5 shows the coherence function for representative CT mediated Jaggregates at a variety of temperatures. With increasing temperature, the coherence function collapses about $r=0$ indicating exciton decoherence and localization. Similar behavior is observed in the Frenkel limit (Fig. 5a) and in the limit of strong Frenkel/CT mixing (Fig. 5b).

The extent of exciton localization can be quantified by the exciton's coherence number. For a linear J-aggregate $N_{\text {Coh }}(T)$ is defined as [31]

$$
N_{C o h}(T) \equiv\left[C^{(e m)}(0, T)\right]^{-1} \sum_{r} C^{(e m)}(r, T)
$$


The coherence number is simply related to the coherence length according to $L_{C o h}=\left(N_{C o h}-1\right) d$ where $d$ is the distance between neighboring molecules. Eq. (16) shows that the coherence number is essentially the "integral" of the peak-normalized coherence function. At low temperature, the exciton is coherently delocalized over the entire aggregate (Fig. 5, black dots) and the coherence function is $N_{C o h}=N$. As the temperature increases, the coherence function localizes and the coherence number decreases until it reaches its lower bound $N_{C o h}=1$ in the limit of infinite temperature.

In Ref. [31] it was shown that in the Frenkel limit, the 0-0 peak intensity is proportional to $N_{\text {coh }}$ for J-aggregates. Indeed, by substituting the wave function of Eq. (6) into Eq. (7) and using Eq. (16) it is straightforward to show that this relation also holds for CT mediated Jaggregates. Since the 0-1 intensity is relatively insensitive to temperature (see Fig. 4) and since in the limit of zero temperature when $N_{c o h}=N$ the ratio is described well by Eq. (12), we modify Eq. (12) to account for thermal effects by replacing $N$ by $N_{c o h}$ :

$$
S_{R}(T) \approx \frac{N_{C o h}(T)}{\langle F E\rangle \lambda_{0}^{2}+(1-\langle F E\rangle)\left(\lambda_{+}+\lambda_{-}\right)^{2}} .
$$

We note that in the Frenkel exciton limit (where $t_{e}$ and $t_{h}$ are both zero to prevent Frenkel/CT mixing) $\langle F E\rangle=1$ and Eq. (17) reduces to $S_{R}(T)=N_{C o h}(T) / \lambda_{0}^{2}$ as previously shown [31]. We further point out that Eq. (17) agrees with a similar form derived in Ref.'s [35,61] for single conjugated polymer chains, in which $S_{R}(T)=\kappa_{0} N_{C o h}(T) / \lambda_{0}^{2}$. Here, we resolve the meaning of the proportionality constant $\kappa_{0}$ as simply a temperature-independent function of primarily the HR factors and FE admixture. Using Eq. (17) we obtain, $\kappa_{0}=\left[\langle F E\rangle+(1-\langle F E\rangle)\left(\lambda_{+}+\lambda_{-}\right)^{2} / \lambda_{0}^{2}\right]^{-1}$.

The accuracy of Eq. (17) is tested in Fig. 6, where the PL ratio as a function of aggregate size is shown for several temperatures. The ratio (open circles) grows linearly with $N$ at T=0K in agreement with Eq. (17), the results of which are represented by the crosses. As the temperature increases, however, the ratio begins to stabilize and level off with increasing $N$, indicating a thermal limit for the PL ratio and hence the coherence size. Again, Eq. (17) agrees well with the numerical results for these higher temperature simulations. 


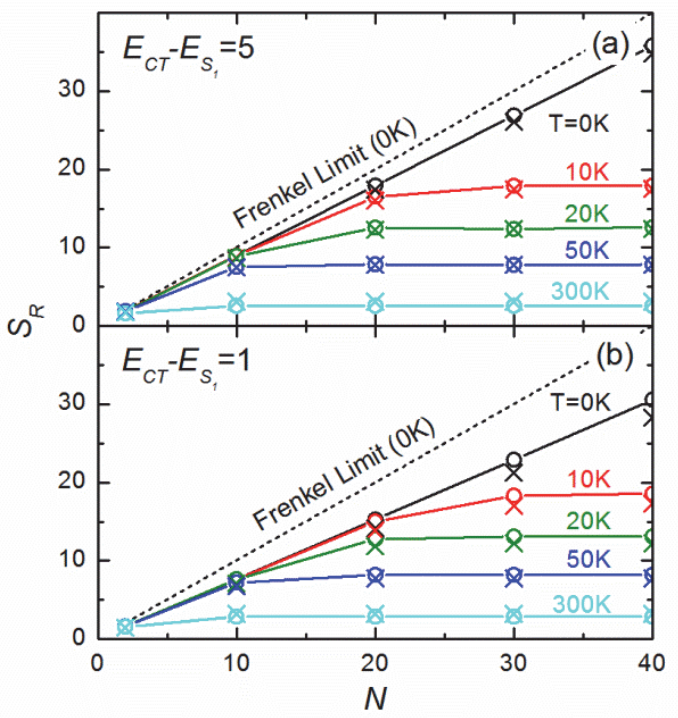

Figure 6. The PL ratio as a function of $N$ at a variety of temperatures for $E_{C T}-E_{S_{1}}=5$ (a) and $E_{C T}-E_{S_{1}}=1$ (b). The remaining system parameters are set so that $\lambda_{0}=$ $1, \lambda_{+}=\lambda_{-}=\sqrt{2} / 2, J_{k}=0$, and $t_{e}=t_{h}=1$ where all energies are given in terms of vibrational quanta. The open circles are calculated numerically while the crosses are determined from Eq. (17) with $\langle F E\rangle$ and $N_{\text {coh }}$ determined numerically. The $T=0 \mathrm{~K}$ results can be compared with the Frenkel limit (black dash) to observe the effect of the FE/CT mixing.

The thermal coherence number appearing in Eq. (17) can be further approximated by assuming a parabolic exciton band with positive curvature. For linear aggregates $(\pi$-stacks) with no energetic disorder, it follows that the thermal coherence number reduces to $[31,35,61]$,

$$
N_{C o h}(T)=\sqrt{\frac{4 \pi \hbar \omega_{c}}{k_{b} T}}
$$

in the thermodynamic limit, i.e. in the limit in which the PL ratio in Fig. 6 levels off. In Eq. (18) $\hbar \omega_{c}$ is the curvature of the exciton band evaluated at the band minimum, with

$$
\left.\omega_{c} \equiv \frac{1}{2} \frac{d^{2} \omega_{\kappa, a=1}}{d \kappa^{2}}\right|_{\kappa=0}
$$

Hence, the main result of this paper, Eq. (17), shows that the PL ratio can be used as a measure of exciton coherence in CT mediated J-aggregates.

\section{Discussion and Conclusion}

Using a Holstein-style Hamiltonian modified to include Frenkel/CT exciton mixing in molecular $\pi$-stacks, we investigated the PL spectral line shape for signatures of exciton 
coherence. The 0-0/0-1 PL ratio was found to be an accurate indicator of the exciton coherence number, $N_{\text {coh }}$, for CT-mediated J-aggregates as was previously established [31] for the more conventional Kasha J-aggregates mediated by Coulombic coupling.[56] We focused on the thermal coherence number, $N_{c o h}(T)$, but our results are expected to hold under other localizing influences such as diagonal or off-diagonal disorder based on the results of Ref.[31].

In the presence of significant wave function overlap, J-aggregates may arise from CTmediated interactions alone (i.e. in the absence of Coulombic coupling) when the electron and hole transfer integrals are in phase, i.e. $t_{e} t_{h}>0$. (Here, translational symmetry is used to define phase).[55] Therefore, in order to emphasize the role of CT-mediated interactions in the PL we have set the Coulomb coupling to zero. However, as long as the band curvature remains positive (J-like), the ratio rule derived here remains valid, even in the more general case where Coulomb coupling and CT-mediated couplings are both present.[46]

The ratio rule applies to any system for which the 0-0 transition from the lowest energy exciton is optically allowed. For example, in the herringbone packing adopted by the oligoacenes such as tetracene and pentacene, the non-parallel alignment of transition dipole moments of translationally inequivalent molecules leads to Davydov splitting, which arises almost entirely from Frenkel/CT mixing.[51,52] In this case, $0-0$ emission from the $b$-polarized lower Davydov component is allowed along $b$. Accordingly, the PL ratio determined by $0-0$ and $0-1$ emission line strengths (both polarized along $b$ ) is an excellent measure of the number of coherently coupled chromophores.[31] We emphasize that the PL ratio is taken with respect to the $0-0$ and 0-1 line strengths. To obtain the line strengths from an actual PL spectrum one must first remove the cubic frequency dependence which arises from the Einstein spontaneous emission rate. Furthermore, in order to extract the intrinsic exciton coherence size from $S_{R}$ one needs to utilize the early-time PL spectrum, obtained before excitons can potentially relax to low-energy defect states.

Determining the coherence number from the PL ratio using Eq. (17) generally requires knowledge of the three HR factors, $\lambda_{0}^{2}, \lambda_{+}^{2}$ and $\lambda_{-}^{2}$ and the Frenkel admixture, $\langle F E\rangle$. The HR factor corresponding to the creation of a local (Frenkel) excitation, $\lambda_{0}^{2}$, is readily obtained from the solution absorption (or PL) spectrum, where the vibronic intensity of the $0-v$ line is proportional to $\lambda_{0}^{2 v} e^{-\lambda^{2}} / v$ !. While $\lambda_{+}$and $\lambda_{-}$are not easy to determine experimentally, the 
calculation of these quantities using quantum chemical software is straightforward.[41,42,62-64] The value of $\langle F E\rangle$ can be readily determined from an analysis of the aggregate absorption spectral line shape as described previously.[46] For the analysis to be valid in practical situations, the $\mathrm{FE}$ admixture should lie approximately in the range $0.5<\langle F E\rangle<1$. When $\langle F E\rangle$ is smaller, the emitting states are dominated by $\mathrm{CT}$ character, and, by virtue of the small oscillator strengths associated with CT states, the PL signal is likely to be weak and difficult to detect. Perhaps more importantly, the higher CT character may lead to excimers, whose broad and unstructured emission may prevent a straightforward evaluation of $S_{R}$.[65-67] Excimer emission also implies the presence of low-frequency intermolecular vibrations which will perturb the intermolecular interactions. Such perturbations could have a non-negligible impact on the exciton coherence which would further complicate analysis of the exciton coherence based on $S_{R}$.

Given the practical range $0.5<\langle F E\rangle<1$ for the Frenkel admixture, Eq. (17) can be used to determine the corresponding range of $N_{\text {Coh }}$ values as,

$$
S_{R}\left[\lambda_{0}^{2}+\left(\lambda_{+}+\lambda_{-}\right)^{2}\right] / 2<N_{C o h}<S_{R} \lambda_{0}^{2} .
$$

From Eq. (20) it can be seen when $\lambda_{+}+\lambda_{-}$approaches $\lambda_{0}$ the range contracts and the association of $N_{\text {Coh }}$ with $\lambda_{0}^{2} S_{R}$ becomes accurate. Indeed, in the special case, $\lambda_{+}+\lambda_{-} \approx \lambda_{0}$, we showed in Appendix A that the ratio $S_{R}$ is exactly, $S_{R}=N / \lambda_{0}^{2}$, at low temperatures (and without disorder) independent of $\langle F E\rangle$. This remarkable result is valid over the entire phase space of Eq. (1) provided only that the exciton band curvature is positive. At higher temperatures (and maintaining $\lambda_{+}+\lambda_{-} \approx \lambda_{0}$ ), the ratio is very accurately given by $S_{R}=N_{c o h} / \lambda_{0}^{2}$. Fortunately, for many systems of interest, the relation $\left(\lambda_{+}+\lambda_{-}\right)^{2} \approx \lambda_{0}^{2}$, approximately holds [42] so that the PL ratio does not deviate much from $N_{c o h} / \lambda^{2}$. For example, in the case of tetracene, the value of $S_{R} \approx 5$ is extracted from experiment at $4 \mathrm{~K}$ [36]. Based on the values of $\lambda_{+}=0.57$ and $\lambda_{-}=0.67$ determined in Ref. [68], the value $\lambda_{0}^{2} \approx 1$ extracted from the solution spectrum, and the value 
$\langle F E\rangle=0.73$ evaluated in Ref. [51], the coherence number using Eq. (17) is estimated to be $N_{C o h} \approx 5.7$, only slightly greater than the value of 5 obtained using $N_{C o h}=\lambda_{0}^{2} S_{R}$. This example illustrates the utility of the ratio formula developed in Ref. [31] for estimating the coherence length even in aggregates dominated by CT interactions. When more accurate values of $N_{c o h}$ are desired, Eq. (17) should be used. In future work we will show that the ratio formula also holds in the presence of multiple intramolecular vibrations, thereby extending its utility as a simple and accurate measure for the exciton coherence number.

Acknowledgements: FCS is supported by the NSF, grant \# DMR-1505437.

\section{Appendix A: Limit of $\lambda_{+}+\lambda_{-}=\lambda_{0}$}

Here we prove that in the special case when $\lambda_{+}+\lambda_{-}=\lambda_{0}$, the low temperature PL ratio is given by $S_{R}=N / \lambda_{0}^{2}$ over the entire parameters space defining the Hamiltonian in Eq. (1).

When $\lambda_{+}+\lambda_{-}=\lambda_{0}$, the totally symmetric $(q=0)$ phonon decouples from the rest of the Hamiltonian. We thus divide the Hamiltonian into two parts,

$$
H=H_{1}+H_{2},
$$

where $H_{1}$ involves only the totally symmetric phonons and $H_{2}$ contains everything else. The Hamiltonian for the totally symmetric phonon is given by

$$
H_{1}=\hbar \omega_{v i b} b_{q=0}^{\dagger} b_{q=0}+\frac{\lambda_{0} \hbar \omega_{v i b}}{\sqrt{N}}\left(b_{q=0}^{\dagger}+b_{q=0}\right)+\frac{\lambda_{0}^{2} \hbar \omega_{v i b}}{N}
$$

which can be simplified to

$$
H_{1}=\hbar \omega_{v i b} \tilde{b}_{q=0}^{\dagger} \tilde{b}_{q=0}
$$


by expressing the totally symmetric phonon operators in terms of the shifted creation and annihilation operators,

$$
\tilde{b}_{q=0}^{\dagger}=b_{q=0}^{\dagger}+\frac{\lambda_{0}}{\sqrt{N}}
$$

and

$$
\tilde{b}_{q=0}=b_{q=0}+\frac{\lambda_{0}}{\sqrt{N}}
$$

The eigenstates of $H_{1}$ are the phonon number states denoted as $\left|\tilde{n}_{q=0}\right\rangle\left(\tilde{n}_{q=0}=0,1,2, \ldots\right)$ with energy $\left\langle\tilde{n}_{q=0}\left|H_{1}\right| \tilde{n}_{q=0}\right\rangle=\tilde{n}_{q=0} \hbar \omega_{v i b}$. Since the $q=0$ phonon is separable from the rest of the Hamiltonian, the number of totally symmetric phonons is a good quantum number of $H$ in Eq. (A1).

The remainder of the Hamiltonian is given by $\mathrm{H}_{2}$,

$$
\begin{aligned}
H_{2} & =E_{S_{1}}+\sum_{k, s}\left[J_{k} \delta_{s, 0}+V_{C T}(s)\left(1-\delta_{s, 0}\right)\right]|k, s\rangle\langle k, s|+\sum_{k, s}\left\{\left(t_{e}+t_{h} e^{i k}\right)|k, s\rangle\langle k, s+1|+H . c .\right\} \\
& +h \omega_{v i b} \sum_{q \neq 0} b_{q}^{\dagger} b_{q}+\frac{\hbar \omega_{v i b}}{\sqrt{N}} \sum_{k, q \neq 0, s}\left\{\left[\lambda_{0} \delta_{s, 0}+\left(\lambda_{-} e^{i q s}+\lambda_{+}\right)\left(1-\delta_{s, 0}\right)\right]|k, s\rangle\langle k+q, s| b_{q}^{\dagger}+H . c .\right\} . \\
& +(1-1 / N) \hbar \omega_{v i b} \lambda_{0}^{2}-2 \lambda_{-} \lambda_{+} \hbar \omega_{v i b} \sum_{k, s}\left(1-\delta_{s, 0}\right)|k, s\rangle\langle k, s|
\end{aligned}
$$

Given that both the number of totally symmetric phonons $\tilde{n}_{q=0}$ and the total momentum $\kappa$ are good quantum numbers of $H$, the eigenstates can be written as

$$
\left|\Psi_{\kappa, \tilde{n}_{q_{0}}, \alpha}\right\rangle=\left|\tilde{n}_{q_{0}}\right\rangle \otimes \sum_{k, s} \sum_{n_{q_{1}}, n_{q_{2}}, \ldots}^{\prime} c_{k, s ; n_{q_{1}}, n_{q_{2}}, \ldots}^{\kappa, \tilde{n}_{q}, \alpha}\left|k, s ; n_{q_{1}}, n_{q_{2}}, \ldots\right\rangle
$$

where we identified the $l$ th phonon wave vector with $q_{l}=2 \pi l / N(l=0,1,2, . . N-1)$. Furthermore, $\alpha(=1,2, \ldots)$ labels the eigenstates with a given $\kappa$ and $\tilde{n}_{q_{0}}$ in order of increasing energy. Note that in contrast to Eq. (6), the totally symmetric phonon has been factored out of Eq. (A7). For a 
J-aggregate, the lowest energy eigenstate is characterized by the quantum numbers $\kappa=0$, $\tilde{n}_{q_{0}}=0$, and $\alpha=1$. At low temperature, this state is the sole source of emitted photons and thus determines the PL spectrum. Inserting Eq. (A7) for $\Psi_{\kappa=0, \tilde{n}_{q_{0}}=0, \alpha=1}$ into Eq. (8) for $v=0$, the line strength from $\Psi_{\kappa=0, \tilde{n}_{q_{0}}=0, \alpha=1}$ to the vibrationless ground state is given by

$$
I_{\kappa=0, \tilde{n}_{q}=0, \alpha=1}^{0-0} \equiv \frac{1}{\mu^{2}}\left|\left\langle g ; v a c|\hat{\mu}| \Psi_{\kappa=0, \tilde{n}_{y=0}=0, \alpha=1}\right\rangle\right|^{2}
$$

which is evaluated as

$$
I_{\kappa=0, \tilde{n}_{00}=0, \alpha=1}^{0-0}=N\left|c_{k=0, s=0 ; 0,0, \ldots}^{\kappa=0, \tilde{n}_{g}=0, \alpha=1}\right|^{2} e^{-\lambda_{0}^{2} / N}
$$

where $e^{-\lambda_{0}^{2} / N}=\left|\left\langle n_{q_{0}}=0 \mid \tilde{n}_{q_{0}}=0\right\rangle\right|^{2}$ is the Franck-Condon factor of the totally symmetric phonon. Similarly the line strength from $\Psi_{\kappa=0, \tilde{n}_{q_{0}}=0, \alpha=1}$ to the ground electronic state with one vibrational quantum $(v=1)$ is given by

$$
I_{\kappa=0, \tilde{n}_{q_{0}}=0, \alpha=1}^{0-1} \equiv \frac{1}{\mu^{2}} \sum_{l}\left|\left\langle g ; 0,1_{q_{l}}, 0, \ldots, 0|\hat{\mu}| \Psi_{\kappa=0, \tilde{n}_{q_{0}}=0, \alpha=1}\right\rangle\right|^{2},
$$

where the sum is over all ground electronic states with a single phonon. Eq. (A10) is further simplified to,

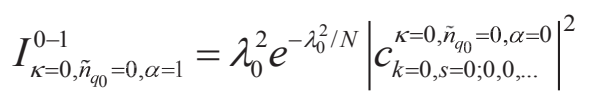

where we have used the Franck-Condon factor, $\left|\left\langle n_{q_{0}}=1 \mid \tilde{n}_{q_{0}}=0\right\rangle\right|^{2}=\lambda_{0}^{2} e^{-\lambda_{0}^{2} / N} / N$. Hence, for the special case of $\lambda_{+}+\lambda_{-}=\lambda_{0}$, the PL ratio at low temperature is given by

$$
\lim _{\lambda_{+}+\lambda_{-} \rightarrow \lambda_{0}} S_{R}(T \rightarrow 0 K)=\frac{I_{\kappa=0, \tilde{n}_{q}=0, \alpha=1}^{0-0}}{I_{\kappa=0, \tilde{n}_{q}=0, \alpha=1}^{0-1}}=\frac{N}{\lambda_{0}^{2}} .
$$

Throughout the manuscript we have assumed that the transition dipole moments of the CT states are negligible. However, in some real systems CT states exhibit non-vanishing 
transition dipole moments. The proof presented above can be extended in a straightforward manner to account for the possibility of finite CT transition dipoles. When considering these transition dipoles, the expectation value of the transition dipole operator is given by $\langle k, s|\hat{\mu}| g\rangle=$ $\sqrt{N} \mu_{s} \delta_{k, 0}$ where $\mu_{s}$ is the transition dipole moment of the electronic state where the electron and hole are separated by $s$ units. Note that transition dipole moment expectation value reduces to the one used above when the transition dipole moments for the CT states are neglected, i.e. $\mu_{|s|>0}=$ 0. Using this relation to solve Eq. (A8) for the 0-0 line strength, Eq. (A9) becomes

$$
I_{\kappa=0, \tilde{n}_{00}=0, \alpha=1}^{0-0}=\frac{N}{\mu_{s=0}^{2}}\left|\sum_{s} \mu_{s} c_{k=0, s ; 0,0, \ldots}^{\kappa=0, \tilde{n}_{0}=0, \alpha=1}\right|^{2} e^{-\lambda_{0}^{2} / N} .
$$

Similarly, solving Eq. (A10) for the 0-1 line strength, Eq. (A11) becomes

$$
I_{\kappa=0, \tilde{n}_{00}=0, \alpha=1}^{0-1}=\frac{\lambda_{0}^{2} e^{-\lambda_{0}^{2} / N}}{\mu_{s=0}^{2}}\left|\sum_{s} \mu_{s} c_{k=0,0, s ; 0, \ldots, \ldots}^{\kappa=0, \tilde{t}_{0}=0, \alpha=1}\right|^{2}
$$

Taking the ratio of Eqs (A13)-(A14), we recover Eq. (A12). Hence, for the special case of $\lambda_{+}+\lambda_{-}=\lambda_{0}$ as $T \rightarrow 0 K$, finite CT transition dipole moments do not affect the PL ratio. We thus expect that even for systems in which the transition dipole moments of the CT states are a significant fraction of the Frenkel transition dipole moment, the ratio will remain an accurate indicator of the exciton's coherence.

\section{Appendix B. The Multi-particle Basis}

Eq. (14) is important, because as an exact theoretical result it can be used to check the accuracy of numerical calculations and the truncation error introduced when an incomplete basis set is used. In Eq. (1) we have represented the basis functions in the delocalized $k, q$ basis in order to facilitate the proof of Eq. (14) in Appendix A. However, the multi-particle basis set [35,69-71] was used for all numerical simulations. In the multi-particle basis set, the basis functions can be classified based on the number of chromophores hosting electronic and vibrational excitations. In the simplest of these states, the electronic and vibrational excitations 
reside on the same chromophore. Such states are referred to as one-particle states. Two-particle Frenkel states consist of one chromophore that is vibronically excited while another chromophore is vibrationally excited. In general, a $n$-particle Frenkel state consisits of one vibronic excitation and $n-1$ vibrational excitations. By contrast, a $n$-particle CT state consists of two vibronic excitations (corresponding to the two ionic states) and $n-2$ vibrational excitations. Hence, there are no CT one-particle states. At the lowest level of approximation only one-particle states are retained in the basis set (note that this approximation precludes CT states) and subsequent improvements involve truncating at an increasingly higher number of particles. For example, in the two-particle approximation all one- and two-particle excitations are retained in the basis set. The two-particle approximation is the lowest level approximation that can account for Frenkel/CT mixing.

In the Frenkel exciton limit, the PL spectrum is generally converged at the two-particle level in the weak to intermediate coupling regimes typical of conjugated organic materials, where the exciton bandwidth is on the order of or smaller than the nuclear relaxation energy. [70] However, as we now show, three-particle states are important for simulating an accurate PL spectrum when there is strong Frenkel/CT mixing. Fig B1 compares the numerically calculated PL ratio as a function of $E_{C T}-E_{S_{1}}$ for an aggregate of ten molecules using the two- and threeparticle approximations. The different panels show the PL ratio for different values of the ionic vibronic coupling parameters. In going from the two- to three-particle approximation, the PL ratio should become closer to the exact value since the basis set is more complete.

While the two-particle approximation approaches the exact result in the Frenkel limit ( $E_{C T} \gg E_{S_{1}}$ ) in Fig. B1(b), it begins to deviate as the diabatic Frenkel and CT excitons come into resonance with one another. This deviation suggests that higher level approximations are necessary to achieve accurate results when there is strong Frenkel/CT mixing. When the threeparticle approximation is used, the numerically calculated PL ratio in Fig. B1(b) agrees nearly perfectly with Eq. (14) which predicts that $S_{R}=N / \lambda_{0}^{2}=10$ over the entire range of $E_{C T}-E_{S_{1}}$. Hence, for the region of phase space we are interested in, the three-particle approximation is sufficient to accurately reproduce the PL ratio for the special case $\lambda_{+}+\lambda_{-}=\lambda$. While we do not have exact results with which to compare the PL ratio for other values of $\lambda_{+}+\lambda_{-}$, the nearly perfect agreement in Fig. B1(b) allows us to be confident that the three-particle approximation 


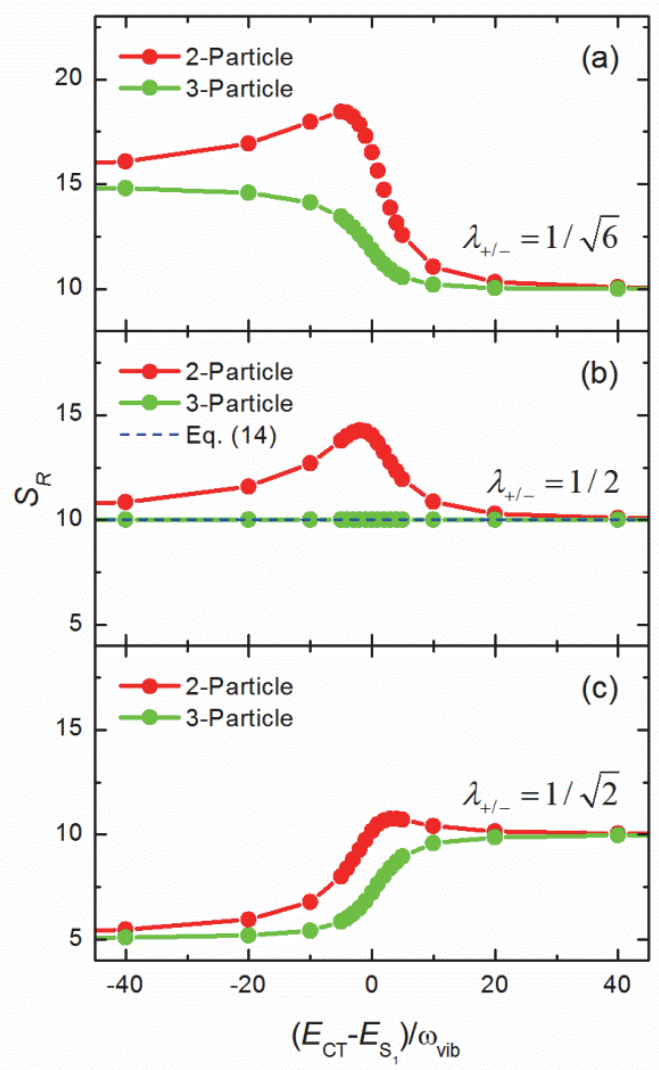

Figure B1. A comparison of the PL ratio as a function of $E_{C T}-E_{S_{1}}$ using the two- and three-particle approximations. In all cases, the system parameters are defined by $\lambda_{0}=1$, $J_{k}=0$, and $t_{e}=t_{h}=1$ where all energies are given in terms of vibrational quanta. The values of the ionic vibronic coupling parameters change in going from panel (a) to (c) and are denoted in each panel. It is seen that the two- and three-particle approximations predict a similar PL ratio in the Frenkel $\left(E_{C T} \gg E_{S_{1}}\right)$ and $\mathrm{CT}\left(E_{C T} \ll E_{S_{1}}\right)$ limits. However, when there is significant Frenkel/CT mixing, the predicted ratios vary significantly between the two approximations. In panel (b) the exact result according to Eq. (14) is also plotted as a check for the convergence for this special case. In panel (b) it is seen that the threeparticle results are essentially converged to the exact value. While we do not know the exact result for panels (a) and (c), the result from (b) gives us confidence that the threeparticle approximation is sufficient for calculating the PL ratio in the region of phase space we are interested in for $\pi$-stacks of conjugated molecules.

will perform equally well when different values of $\lambda_{+/-}$relevant to conjugated organic systems are used. All numerical results contained in this paper for systems having $N>2$ were calculated using the three-particle approximation. For the dimer simulations, the two-particle approximation, which forms the complete basis set, was used.

\section{References}

[1] S. Gelinas, A. Rao, A. Kumar, S.L. Smith, A.W. Chin, J. Clark, T.S. van der Poll, G.C. Bazan, R.H. Friend, Science 343 (2014) 512.

[2] L.G. Kaake, D. Moses, A.J. Heeger, J. Phys. Chem. Lett. 4 (2013) 2264. 
[3] L.G. Kaake, J.J. Jasieniak, R.C. Bakus, G.C. Welch, D. Moses, G.C. Bazan, A.J. Heeger, J. Am. Chem. Soc. 134 (2012) 19828.

[4] N. Banerji, J. Mater. Chem. C 1 (2013) 3052.

[5] S. Mukamel, J. Phys. Chem A 117 (2013) 10563.

[6] Y. Song, S.N. Clafton, R.D. Pensack, T.W. Kee, G.D. Scholes, Nat Commun 5 (2014).

[7] E.R. Bittner, C. Silva, Nat Commun 5 (2014).

[8] I. Kassal, J. Yuen-Zhou, S. Rahimi-Keshari, The Journal of Physical Chemistry Letters 4 (2013) 362 .

[9] J. Strümpfer, M. Şener, K. Schulten, J. Phys. Chem. Lett. 3 (2012) 536.

[10] I. Hwang, G.D. Scholes, Chemistry of Materials 23 (2011) 610.

[11] J.E. Donehue, O.P. Varnavski, R. Cemborski, M. Iyoda, I. Goodson, T., J. Am. Chem. Soc. 133 (2011) 4819.

[12] G. Panitchayangkoon, D. Hayes, K.A. Fransted, J.R. Caram, E. Harel, J.Z. Wen, R.E. Blankenship, G.S. Engel, Proc. Nat. Acad. Sci. USA 107 (2010) 12766.

[13] G.S. Engel, T.R. Calhoun, E.L. Read, T.K. Ahn, T. Mancal, Y.C. Cheng, R.E. Blankenship, G.R. Fleming, Nature 446 (2007) 782.

[14] M. Dahlbom, T. Pullerits, S. Mukamel, V. Sundstrom, J. Phys. Chem. B 105 (2001) 5515.

[15] M. Chachisvilis, O. Kuhn, T. Pullerits, V. Sundstrom, Journal of Physical Chemistry B 101 (1997) 7275.

[16] V. Chernyak, T. Meier, E. Tsiper, S. Mukamel, J. Phys. Chem. A 103 (1999) 10294.

[17] R. Monshouwer, M. Abrahamsson, F. vanMourik, R. vanGrondelle, J. Phys. Chem. B 101 (1997) 7241.

[18] M. Muller, A. Paulheim, A. Eisfeld, M. Sokolowski, J. Chem. Phys. 139 (2013) 044302.

[19] M. Voigt, A. Langner, P. Schouwink, J.M. Lupton, R.F. Mahrt, M. Sokolowski, J. Chem. Phys. 127 (2007) 114705.

[20] E.O. Potma, D.A. Wiersma, J. Chem. Phys. 108 (1998) 4894.

[21] S. Deboer, D.A. Wiersma, Chem. Phys. Lett. 165 (1990) 45.

[22] H. Fidder, J. Knoester, D.A. Wiersma, Chem. Phys. Lett. 171 (1990) 529.

[23] E.W. Knapp, Chem. Phys. 85 (1984) 73.

[24] H. Fidder, J. Knoester, D.A. Wiersma, J. Chem. Phys. 95 (1991) 7880. 
[25] O. Kuhn, V. Sundstrom, J. Chem. Phys. 107 (1997) 4154.

[26] T. Pullerits, M. Chachisvilis, V. Sundstrom, J. Phys. Chem. 100 (1996) 10787.

[27] J. Knoester, J. Chem. Phys. 99 (1993) 8466.

[28] T. Kobayashi (Ed.)^(Eds.), J-aggregates. World Scientific, Singapore, 1996.

[29] F. Wurthner, T.E. Kaiser, C.R. Saha-Moller, Angew. Chem. Int. Ed. 50 (2011) 3376

[30] G. Scheibe, Kolloid-Zeitschrift 82 (1938) 1.

[31] F.C. Spano, H. Yamagata, J. Phys. Chem. B 115 (2011) 5133.

[32] R. Tempelaar, F.C. Spano, J. Knoester, T.L.C. Jansen, J. Phys. Chem. Lett. 5 (2014) 1505.

[33] J. Sung, P. Kim, B. Fimmel, F. Wurthner, D. Kim, Nat. Commun. 6 (2015) 8646.

[34] F. Dubin, R. Melet, T. Barisien, R. Grousson, L. Legrand, M. Schott, V. Voliotist, Nature Physics 2 (2006) 32.

[35] H. Yamagata, F.C. Spano, J. Chem. Phys 135 (2011) 054906.

[36] S.-H. Lim, T.G. Bjorklund, F.C. Spano, C.J. Bardeen, Phys. Rev. Lett. 92 (2004) 107402.

[37] S. Tanaka, K. Miyata, T. Sugimoto, K. Watanabe, T. Uemura, J. Takeya, Y. Matsumoto, J. Phys. Chem. C (2016).

[38] B. Khachatryan, T.D. Nguyen, Z.V. Vardeny, E. Ehrenfreund, Phys. Rev. B 86 (2012) 195203.

[39] M. Hoffmann, Z.G. Soos, Phys. Rev. B 66 (2002) 024305.

[40] M. Hoffmann, K. Schmidt, T. Fritz, T. Hasche, V.M. Agranovich, K. Leo, Chem. Phys. $258(2000) 73$.

[41] L. Gisslen, R. Scholz, Phys. Rev. B 83 (2011) 155311.

[42] L. Gisslen, R. Scholz, Phys. Rev. B 80 (2009) 115309.

[43] V. Settels, A. Schubert, M. Tafipolski, W. Liu, V. Stehr, A.K. Topczak, J. Pflaum, C. Deibel, R.F. Fink, V. Engel, B. Engels, J. Am. Chem. Soc. 136 (2014) 9327.

[44] A. Schubert, V. Settels, W. Liu, F. Würthner, C. Meier, R.F. Fink, S. Schindlbeck, S. Lochbrunner, B. Engels, V. Engel, J. Phys. Chem. Lett. 4 (2013) 792.

[45] R.F. Fink, J. Seibt, V. Engel, M. Renz, M. Kaupp, S. Lochbrunner, H.M. Zhao, J. Pfister, F. Wurthner, B. Engels, J. Am. Chem. Soc. 130 (2008) 12858.

[46] N.J. Hestand, F.C. Spano, J. Chem. Phys. 143 (2015) 244707. 
[47] H. Yamagata, D.S. Maxwell, J. Fan, K.R. Kittilstved, A.L. Briseno, M.D. Barnes, F.C. Spano, J. Phys. Chem. C 118 (2014) 28842.

[48] N.J. Hestand, R. Tempelaar, J. Knoester, T.L.C. Jansen, F.C. Spano, Phys. Rev. B 91 (2015) 195315

[49] B. Petelenz, P. Petelenz, H.F. Shurvell, V.H. Smith, Chem. Phys. 119 (1988) 25.

[50] R. Schuster, M. Knupfer, H. Berger, Phys. Rev. Lett. 98 (2007) 037402.

[51] H. Yamagata, J. Norton, E. Hontz, Y. Olivier, D. Beljonne, J.L. Bredas, R.J. Silbey, F.C. Spano, J. Chem. Phys. 134 (2011) 204703.

[52] N.J. Hestand, H. Yamagata, B.L. Xu, D.Z. Sun, Y. Zhong, A.R. Harutyunyan, G.G. Chen, H.L. Dai, Y. Rao, F.C. Spano, J. Phys. Chem. C 119 (2015) 22137.

[53] X.Y. Zhu, Q. Yang, M. Muntwiler, Acc. Chem. Res. 42 (2009) 1779.

[54] M.B. Smith, J. Michl, Ann. Rev. Phys. Chem. 64 (2013) 361.

[55] H. Yamagata, C.M. Pochas, F.C. Spano, J. Phys. Chem. B 116 (2012) 14494.

[56] M. Kasha, Radiation Research 20 (1963) 55.

[57] F.C. Spano, Acc. Chem. Res. 43 (2010) 429.

[58] R.E. Merrifield, J. Chem. Phys. 34 (1961) 1835.

[59] D. Beljonne, H. Yamagata, J.L. Bredas, F.C. Spano, Y. Olivier, Phys. Rev. Lett. 110 (2013) 226402.

[60] F.C. Spano, J. Chem. Phys. 116 (2002) 5877.

[61] H. Yamagata, F.C. Spano, J. Phys. Chem. Lett. (2014).

[62] J.L. Bredas, D. Beljonne, V. Coropceanu, J. Cornil, Chem. Rev. 104 (2004) 4971.

[63] J. Gierschner, H.-G. Mack, L. Lüer, D. Oelkrug, The Journal of Chemical Physics 116 (2002) 8596.

[64] D. Braun, B.C. Titeca, A. Ceulemans, Journal of Porphyrins and Phthalocyanines 5 (2001) 33.

[65] J.M. Lim, P. Kim, M.C. Yoon, J. Sung, V. Dehm, Z.J. Chen, F. Wurthner, D. Kim, Chem. Sci. 4 (2013) 388.

[66] J.M. Giaimo, J.V. Lockard, L.E. Sinks, A.M. Scott, T.M. Wilson, M.R. Wasielewski, J. Phys. Chem. A 112 (2008) 2322.

[67] E.E. Neuteboom, S.C. Meskers, E. Meijer, R.A. Janssen, Macromol. Chem. Phys. 205 (2004) 217. 
[68] V. Coropceanu, M. Malagoli, D.A. da Silva Filho, N.E. Gruhn, T.G. Bill, J.L. Brédas, Phys. Rev. Lett. 89 (2002) 275503.

[69] M.R. Philpott, J. Chem. Phys. 55 (1971) 2039.

[70] F.C. Spano, J. Chem. Phys. 116 (2002) 5877.

[71] A. Stradomska, P. Petelenz, J. Chem. Phys. 131 (2009) 044507. 